\title{
Probing the $\mu$-from- $\nu$ supersymmetric standard model with displaced multileptons from the decay of a Higgs boson at the LHC
}

\author{
Pradipta Ghosh, ${ }^{1,2, *}$ Daniel E. López-Fogliani, ${ }^{3, \dagger}$ Vasiliki A. Mitsou, ${ }^{4, \ddagger}$ Carlos Muñoz, ${ }^{1,2, \S}$ and Roberto Ruiz de Austri ${ }^{4, \|}$ \\ ${ }^{1}$ Departamento de Física Teórica, Universidad Autónoma de Madrid, Cantoblanco, 28049 Madrid, Spain \\ ${ }^{2}$ Instituto de Física Teórica UAM-CSIC, Campus de Cantoblanco UAM, 28049 Madrid, Spain \\ ${ }^{3}$ Departamento de Física, Universidad de Buenos Aires and IFIBA-CONICET, 1428 Buenos Aires, Argentina \\ ${ }^{4}$ Instituto de Física Corpuscular CSIC-UV, c/ Catedrático José Beltrán, 2, 46980 Paterna (Valencia), Spain
}

(Received 19 December 2012; revised manuscript received 29 March 2013; published 8 July 2013)

The " $\mu$ from $\nu$ " supersymmetric standard model ( $\mu \nu \mathrm{SSM}$ ) cures the $\mu$ problem and concurrently reproduces measured neutrino data by using a set of usual right-handed neutrino superfields. Recently, the LHC has revealed the first scalar boson which naturally makes it tempting to test $\mu \nu \mathrm{SSM}$ in the light of this new discovery. We show that this new scalar, while decaying to a pair of unstable long-lived neutralinos, can lead to a distinct signal with nonprompt multileptons. With concomitant collider analysis we show that this signal provides an intriguing signature of the model, pronounced with light neutralinos. Evidence of this signal is well envisaged with sophisticated displaced vertex analysis, which deserves experimental attention.

DOI: 10.1103/PhysRevD.88.015009

The $\mu \nu \mathrm{SSM}[1,2]$ contains, in the superpotential $W$, in addition to the Yukawa couplings for quarks and charged leptons of the minimal supersymmetric standard model (MSSM) [3], Yukawas for neutrinos and two additional types of terms involving the Higgs doublet superfields, $\hat{H}_{u}$ and $\hat{H}_{d}$, and the three right-handed neutrino superfields $\hat{\nu}_{i}^{c}[1,2]$ :

$$
\begin{aligned}
W= & \epsilon_{a b}\left(Y_{u_{i j}} \hat{H}_{u}^{b} \hat{Q}_{i}^{a} \hat{u}_{j}^{c}+Y_{d_{i j}} \hat{H}_{d}^{a} \hat{Q}_{i}^{b} \hat{d}_{j}^{c}+Y_{e_{i j}} \hat{H}_{d}^{a} \hat{L}_{i}^{b} \hat{e}_{j}^{c}\right. \\
& \left.+Y_{\nu_{i j}} \hat{H}_{u}^{b} \hat{L}_{i}^{a} \hat{\nu}_{j}^{c}-\lambda_{i} \hat{\nu}_{i}^{c} \hat{H}_{d}^{a} \hat{H}_{u}^{b}\right)+\frac{1}{3} \kappa_{i j k} \hat{\nu}_{i}^{c} \hat{\nu}_{j}^{c} \hat{\nu}_{k}^{c} .
\end{aligned}
$$

The simultaneous presence of the last three terms in Eq. (1) gives rise to explicit breaking of $R$ parity $\left(R_{p}\right)$. With only dimensionless trilinear couplings in $W$, the electroweak (EW) scale arises through the soft supersymmetry (SUSY)-breaking terms in the scalar potential. Thus all known particle physics phenomenology can be reproduced in the $\mu \nu \mathrm{SSM}$ with only one scale. Once the EW symmetry is spontaneously broken, the neutral scalars develop, in general, the following vacuum expectation values (VEVs): $\left\langle H_{d}^{0}\right\rangle=v_{d},\left\langle H_{u}^{0}\right\rangle=v_{u},\left\langle\tilde{\nu}_{i}\right\rangle=\nu_{i},\left\langle\tilde{\nu}_{i}^{c}\right\rangle=\nu_{i}^{c}$. An effective interaction $\mu \hat{H}_{d} \hat{H}_{u}$, with $\mu \equiv \lambda_{i} \nu_{i}^{c}$, is generated through the fifth term of Eq. (1), solving the $\mu$ problem of the MSSM [4] without introducing an extra singlet superfield as in the case of the next-to-MSSM (NMSSM) [5]. The sixth term in Eq. (1) avoids the existence of a Goldstone boson. It also generates EW-scale effective Majorana masses $\left(2 \kappa \nu_{i}^{c}\right)$ for right-handed neutrinos, which give

\footnotetext{
*pradipta.ghosh@uam.es

†daniel.lopez@df.uba.ar

*vasiliki.mitsou@ific.uv.es

§carlos.munnoz@uam.es

"rruiz@ific.uv.es
}

PACS numbers: 12.60.Jv, 12.60.Fr, 14.80.Da, 14.80.Ly

rise to a TeV-scale seesaw with $Y_{\nu} \sim 10^{-6}$ (like $Y_{e}$ ) and, together with $R_{p}$ violation $\left(\not R_{p}\right)$, are instrumental in reproducing the measured neutrino mass squared differences and mixing angles $[2,6-8]$ at the tree level. This feature is unlike the bilinear $\not_{p}$ model [9] where only one mass is generated at the tree level and loop corrections are necessary to generate at least a second mass and a neutrino mixing matrix compatible with experiments. In the bilinear model, the $\mu$-like problem [10] is also augmented with three bilinear terms.

In the $\mu \nu \mathrm{SSM}$ as a consequence of the $\not R_{p}$, all the neutral fermions (scalars) mix together and there are 10 neutralino (8 $C P$-even and $7 C P$-odd) mass eigenstates. Analyses of the $\mu \nu \mathrm{SSM}$, with attention to the neutrino and LHC phenomenology, have also been addressed in [2,7,8,11-14]. Other analyses concerning cosmology such as gravitino dark matter and electroweak baryogenesis can be found in $[15,16]$, respectively.

Thus the $\mu \nu \mathrm{SSM}$ is a well-motivated SUSY model with enriched phenomenology and notable signatures, which definitely deserve rigorous analyses by the LHC Collaboration. However, although SUSY searches remain one of the primary targets for the LHC, the discovery of a new scalar boson with a mass around $125 \mathrm{GeV}$ by the ATLAS [17] and CMS [18] collaborations has attracted the attention of the community. In spite of the observed decay rates of this particle being compatible with those of the standard model (SM) Higgs boson, a departure from the SM predictions remains a possibility since new LHC data are being analyzed.

We present a dedicated collider analysis together with detector simulation of an intriguing signal in the $\mu \nu \mathrm{SSM}$ featuring nonprompt multileptons at the LHC, arising from the beyond SM decay of a $125 \mathrm{GeV}$ scalar into a pair of lightest neutralinos $\left(\tilde{\chi}^{0}\right)$. Since $R_{p}$ is broken, each $\tilde{\chi}^{0}$ 
decays into a scalar/pseudoscalar $(h / P)$ and a neutrino $(\nu)$, with the $h / P$ further driven to decay into $\tau^{+} \tau^{-}$, giving rise to a $4 \tau$ final state. Because of the value of $Y_{\nu}, \not R_{p}$ is small and $\tilde{\chi}^{0}$ decay leads to a displaced vertex (DV). We investigate the situation when the $\tilde{\chi}^{0}$ decays inside the inner tracker, thereby yielding clean detectable signatures. Although $h / P \rightarrow b \bar{b}$ is dominant over a broad range of parameters, we stick to $2 m_{\tau} \lesssim m_{h / P} \lesssim 2 m_{b}$, which allows us to demonstrate two possible signatures characterized by (i) high lepton multiplicity and (ii) charged tracks originating from DVs, which can be explored through distinct experimental approaches.

It remains to elucidate, if a possible excess of fourlepton ( $e, \mu$ coming from $\tau$-decay) events is observed at the LHC, how to distinguish the $\mu \nu \mathrm{SSM}$ from other models. A dedicated measurement of the displaced charged tracks can reject all possible similar final states with prompt leptons. In bilinear $\not_{p}$ models with minimal superfield content, $\tilde{\chi}^{0}$ with mass below $m_{W}$ dominantly decays through $\ell W^{*} / \nu Z^{*}$ with $\ell=e, \mu, \tau$, while the decay length $\left(l_{D L}\right)$ scales as $1 / m_{\tilde{\chi}^{0}}^{4}$. In the $\mu \nu \mathrm{SSM}$, however, available lighter singlet like $h / P$ states provide new two-body decay modes, $\tilde{\chi}^{0} \rightarrow h / P+\nu$, which can reduce $l_{D L}$ by orders of magnitude, as hinted in [11]. This feature manifests notably in the regime $m_{\tilde{\chi}^{0}}<20 \mathrm{GeV}$ where bilinear $\not R_{p}$ models predict $l_{D L} \sim 100 \mathrm{~m}$ [11], beyond detector coverage, and thus mimic the known missing energy $\left(\mathscr{E}_{T}\right)$ signature with conserved $R_{p}$. On the other hand, models with a trilinear $\not R_{p}$ term, for example $\hat{L}_{i} \hat{L}_{j} \hat{E}_{k}^{c}$ [9], can produce a moderate to large displaced vertex $(1 \mathrm{~cm}-3 \mathrm{~m})$ for $m_{\tilde{\chi}^{0}}<20 \mathrm{GeV}$. Such a light $\tilde{\chi}^{0}$ within minimal SUSY models will, however, give rise to a high branching ratio for a SM-like Higgs $\rightarrow \tilde{\chi}^{0} \tilde{\chi}^{0}$ decay mode. Since resulting final states contain visible particles, this scenario is tightly constrained from experimental data. This problem is, however, ameliorated in the $\mu \nu \mathrm{SSM}$ since the said branching fraction can still remain suppressed due to the predominant singlet composition of $\tilde{\chi}^{0}$.

Wrapping up, moderately displaced $(\geq 1 \mathrm{~cm})$ yet detectable charged tracks from a light $\tilde{\chi}^{0}$, as in the $\mu \nu \mathrm{SSM}$, are hardly possible with other minimal SUSY models with or without $R_{p}$. Nonminimal SUSY models with or without $\not R_{p}$ can, however, produce similar displaced final states. As an example, in the NMSSM these states can appear when a light next-to-lightest supersymmetric particle (produced directly or from Higgs decay) decays to a very light LSP (to yield small $\mathscr{E}_{T}$ ) and a light scalar/pseudoscalar. This scenario in reality, however, is hardly a realistic choice, with the experimental measurement of the invisible Z-decay width and other LEP (and LHC) measurements. On the contrary, nonminimal SUSY models with $\not R_{p}$ produce an irreducible imposter for this specific signal. Nevertheless, there exist other unique decay modes, for example, multilepton final states from long Higgs-to-Higgs cascades [13], which can provide a distinctive signal of the $\mu \nu \mathrm{SSM}$ because more Higgses are present. It is also worth remarking in this context that whereas the trilinear $\not R_{p}$ terms in the $\mu \nu \mathrm{SSM}$ are useful for reproducing neutrino data at tree level and for solving the $\mu$ problem, trilinear $\not R_{p}$ terms such as $\hat{L}_{i} \hat{L}_{j} \hat{E}_{k}^{c}$ do not attempt to solve the $\mu$ problem and generate neutrino masses only through loops.

The noticeable footprint of the $\mu \nu \mathrm{SSM}$ relies on the presence of light $\tilde{\chi}^{0}$ and lighter $h, P$, which are experimentally feasible when predominantly singlet in nature. Concealing the complex flavor structure and neglecting terms $\propto Y_{\nu}, \nu$ for smallness, $m_{\tilde{\chi}^{0}} \sim 2 \kappa \nu^{c}$, for $\left|2 \kappa \nu^{c}\right| \ll$ $|\mu|,\left|m_{\text {gaugino }}\right|$, which is favored with small $\kappa$. Also, in the limit of moderately small $\lambda(\sim 0.1)$ singlets like $h, P$ are already decoupled from the doublet sector, and one gets from Ref. [2] $m_{h}^{2} \sim m_{\tilde{\chi}^{0}}^{2}+m_{\tilde{\chi}^{0}} A_{\kappa} / 2$ and $m_{P}^{2} \sim-3 m_{\tilde{\chi}^{0}} A_{\kappa} / 2$. In the small $\lambda$ limit, $\nu^{c} \sim 1 \mathrm{TeV}$ is also apparent as $\mu\left(\sim 3 \lambda \nu^{c}\right) \gtrsim 100 \mathrm{GeV}$ from chargino searches. Thus, in the region of interest, that is, $2 m_{\tau} \lesssim\left|m_{\tilde{\chi}^{0}}\right| \lesssim 20 \mathrm{GeV}$, naively $10^{-3} \lesssim|\kappa| \lesssim 10^{-2}$, and from the constraint $2 m_{\tau} \lesssim m_{P} \lesssim 2 m_{b}$ one also obtains $0.4 \lesssim\left|A_{\kappa}\right| \lesssim$ $30 \mathrm{GeV}$. Among other relevant parameters, small $\tan \beta$ seems useful to evade LEP constraints. The aforesaid discussion is an artifact of the nonminimal nature of the $\mu \nu \mathrm{SSM}$ and thus could be related to well-studied [19,20] corners of the NMSSM parameter space with a similar spectrum. More precisely, a light $P$ in the NMSSM is related to a solution of the little hierarchy problem by using small $A_{\kappa}$ [20]. Light $\tilde{\chi}^{0}$ and $h$, on the other hand, are related to the revival of an approximate Peccei-Quinn symmetry of the NMSSM in the limit of vanishing $\kappa$ $[19,20]$. Although the spectrum of the $\mu \nu \mathrm{SSM}$ is grossly enriched with three singlet $\hat{\nu}_{i}^{c}$ and $\not_{p}$, the presence of light $h, P$ and $\tilde{\chi}^{0}$ in the model is still well motivated by the nonminimal nature. This region of parameter space also contains a clear fingerprint of the $\mu \nu \mathrm{SSM}$ through detectable DVs for a very light $\tilde{\chi}^{0}$.

In what follows, neutralino mass eigenstates are denoted by $\tilde{\chi}_{1, \ldots, 10}^{0}$, where $\tilde{\chi}_{i}^{0}$, with $i=1,2,3$, coincide with the three left-handed neutrinos. $\tilde{\chi}_{4}^{0}$ has been identified as the lightest neutralino, with leading right-handed neutrino composition at the limit of small $\kappa$. $C P$-even ( $C P$-odd) scalar mass eigenstates are denoted by $h_{1, \ldots, 8}\left(P_{1, \ldots, 7}\right)$. In the chosen benchmark, $h_{4}$ is basically decoupled from the right-handed sneutrinos $\tilde{\nu}_{i}^{c}$ and is the lightest doublet-like Higgs. Three lightest scalar $h_{i}$ (pseudoscalar $P_{i}$ ) states are the ones composed mainly of $\tilde{\nu}_{i}^{c}$.

As in Ref. [2], we eliminate eight soft masses in favor of the corresponding VEVs. We choose $\nu_{i}^{c}=780 \mathrm{GeV}$, $\tan \beta \approx \frac{v_{u}}{v_{d}}=3.7$ and $v \approx \sqrt{v_{u}^{2}+v_{d}^{2}} \approx 174 \mathrm{GeV}$. We assume gaugino mass unification at the grand unified theory scale and consider $M_{2}=500 \mathrm{GeV}$ at the EW scale. We have fixed the following universal soft parameters at 
low energy: $m_{\tilde{e}^{c}}=m_{\tilde{u}^{c}}=m_{\tilde{d}^{c}}=m_{\tilde{Q}}=1 \mathrm{TeV}, A_{\lambda}=$ $990 \mathrm{GeV}, A_{\kappa}=5 \mathrm{GeV}, A_{e}=A_{d}=-A_{\nu}=1 \mathrm{TeV}$ and $A_{u}=2.4 \mathrm{TeV}$. Chosen values for $m_{\tilde{Q}}$ and $A_{u}$ are crucial for sizable loop corrections to reach $m_{h_{4}} \sim 125 \mathrm{GeV}$ with selected $\lambda_{i}$. At the limit of moderately small $\lambda$ (and small $\kappa)$, the value of $A_{\lambda}$ is relevant for doublet-singlet mixing. We have checked that for the chosen values of the parameters, $A_{\lambda}$ can be varied in the range $980 \lesssim A_{\lambda} \lesssim 1040 \mathrm{GeV}$ without changing the studied signal significantly. The remaining soft parameters can be arbitrarily changed without altering significantly the discussion presented here.

The values of the low-energy dimensionless free parameters that we assume are $\lambda_{i}=0.11, \kappa_{111}=-0.0073$, $\kappa_{222}=-0.0075, \kappa_{333}=-0.0077$, setting all other $\kappa_{i j k}$ to zero. $\kappa_{i i i}$ are taken to be pseudodegenerate for simplicity. With universal $\kappa_{i i i}$, only one linear combination of the right-handed neutrinos mixes in an efficient way with the MSSM neutralinos. Then, depending on the sign of $\kappa, \tilde{\chi}_{4}^{0}$ or $\tilde{\chi}_{6}^{0}$ will have a significant MSSM neutralino component. In our benchmark point where $m_{\tilde{\chi}_{4}^{0}} \approx 9.6 \mathrm{GeV}, m_{\tilde{\chi}_{5}^{0}} \approx$ $11.5 \mathrm{GeV}, m_{\tilde{\chi}_{6}^{0}} \approx 11.9 \mathrm{GeV}$, the MSSM neutralino admixture is sizable $(\sim 2 \%)$ in $\tilde{\chi}_{4}^{0}$ and we get $\operatorname{Br}\left(h_{4} \rightarrow \tilde{\chi}_{4}^{0} \tilde{\chi}_{4}^{0}\right) \approx$ $6 \%$. If $\kappa_{i i i}>0$ were selected instead, then $h_{4}$ would decay mainly to $\tilde{\chi}_{6}^{0} \tilde{\chi}_{6}^{0}$, followed by fast decays $\tilde{\chi}_{6}^{0} \rightarrow \tilde{\chi}_{4,5}^{0} \mu^{+} \mu^{-}$, $\tilde{\chi}_{5}^{0} \rightarrow \tilde{\chi}_{4}^{0} \mu^{+} \mu^{-}$. Since the produced muons are soft, they are difficult to trigger due to their very low transverse momentum, $p_{\mathrm{T}}$. Thus we stick to $\kappa_{i i i}<0$, so that the relevant signal would be produced by the $h_{4} \rightarrow \tilde{\chi}_{4}^{0} \tilde{\chi}_{4}^{0}$ decay. In the scalar sector relevant masses are $m_{P_{1}} \approx 3.6 \mathrm{GeV}, m_{P_{2}} \approx 3.8 \mathrm{GeV}, m_{P_{3}} \approx 5.5 \mathrm{GeV}$, and $m_{h_{1}} \approx$ $7.5 \mathrm{GeV}, m_{h_{2}} \approx 8.0 \mathrm{GeV}, m_{h_{3}} \approx 19.6 \mathrm{GeV}$ with $m_{h_{4}} \approx$ $125.7 \mathrm{GeV}$. Consequently, $\tilde{\chi}_{4}^{0}$ decays to $P_{i}+\nu$ are favorable with larger available phase space. Although two-body decays of $\tilde{\chi}_{4}^{0}$ are kinematically possible, we have nevertheless computed the three-body decays for greater accuracy. With the chosen mass spectrum, $\operatorname{Br}\left(\tilde{\chi}_{4}^{0} \rightarrow\right.$ $\left.\sum_{i=1}^{3} \tilde{\chi}_{i}^{0} \tau^{+} \tau^{-}\right) \approx 99 \%$, while the remaining $1 \%$ is shared between $\tilde{\chi}_{i}^{0} \mu^{+} \mu^{-}$and $\tilde{\chi}_{i}^{0} q_{j} \bar{q}_{j}$. Thus, the schematic $h_{4}$ decay chain studied is $h_{4} \rightarrow \tilde{\chi}_{4}^{0} \tilde{\chi}_{4}^{0} \rightarrow 2 h_{i}^{*} / P_{i}^{*} 2 \nu \rightarrow$ $2 \tau^{+} 2 \tau^{-} 2 \nu$.

The matrix $Y_{\nu_{i j}}$ and the VEVs of the left-handed sneutrinos, $\nu_{i}$, are connected to the reproduction of neutrino physics. The processes $h_{i} / P_{i} \rightarrow \ell^{+} \ell^{-}$, on the other hand, are, to a very good approximation, independent of $Y_{\nu_{i j}}$ (and $\nu_{i}$ ). Hence, multiplicity of the charged leptons in the process is practically independent of $\nu_{i}$ and $Y_{\nu_{i j}}$. As a corollary, a range for $Y_{\nu_{i j}}$ and $\nu_{i}$ predicting correct neutrino physics is well anticipated without drastic alteration in the event topology with displaced multileptons.

For the studied benchmark point neutrino-sector parameters have been chosen to result in $m_{\tilde{\chi}_{3}^{0}} \sim$ $4.9 \times 10^{-11} \mathrm{GeV}$, giving a decay width $\Gamma_{\tilde{\chi}_{4}^{0}} \approx 6.7 \times$ $10^{-16} \mathrm{GeV}^{-1}$, which corresponds to a proper lifetime $\tau_{\tilde{\chi}_{4}^{0}} \approx 10^{-9} \mathrm{~s}$. One can avail the underlying relation between neutrino physics and $\tilde{\chi}_{4}^{0}$ decay kinematics in the $\mu \nu \mathrm{SSM}$ through a common set of parameters $Y_{\nu}, \kappa, \lambda, \nu^{c}$, gaugino masses, etc. [11,12], to obtain shorter $\tau_{\tilde{\chi}_{4}^{0}}$ through an increase in the absolute neutrino mass scale. Another viable handle to modify $\tau_{\tilde{\chi}_{4}^{0}}$ is through a change in $\tilde{\chi}_{4}^{0}$ composition, namely, by altering the relative dominance of gaugino masses, the right-handed neutrino Majorana mass, $2 \kappa \nu^{c}$ and the $\mu$ parameter, $3 \lambda \nu^{c}$ [7,11,12]. We have checked that variations in the benchmark point do not induce significant changes in the lepton multiplicity, but do affect $\Gamma_{\tilde{\chi}_{4}^{0}}$, i.e. $\tau_{\tilde{\chi}_{4}^{0}}$, which remains in the experimentally accessible range of $\mathrm{mm}$ to $\mathrm{m}$.

For numerical studies, PYTHIA (6.4.09) [21] has been used as the MC event generator with a default parton distribution function at $8 \mathrm{TeV}$ center-of-mass energy $(\sqrt{s})$. The renormalization/factorization scale is set equal to the parton-level $\sqrt{s}$. The initial and final state radiation and multiple interactions are kept switched on. The mass spectrum and decays are computed with a customdeveloped code. For the SM-like $h_{4}$, the $g g \rightarrow h_{4}$ cross section $(6.51 \mathrm{pb})$ is rescaled by the reduced coupling [13], yielding a next-to-next-to leading order $g g \rightarrow h_{4}$ production cross section of $19.3 \mathrm{pb}$ [22]. PYTHIA outputs are passed through PGS4 [23] to simulate the detector response. All resulting distributions have been rescaled to an expected integrated luminosity of $\mathcal{L}=20 \mathrm{fb}^{-1}$ corresponding to the full 2012 data set.

The $\mu \nu \mathrm{SSM}$ is characterized by the production of several high- $p_{\mathrm{T}}$ leptons, as demonstrated in Fig. 1 (top), where the $e, \mu$ and hadronically decaying $\tau\left(\tau^{\mathrm{had}}\right)$ multiplicity distributions are drawn for leptons with $p_{\mathrm{T}}>$ $10 \mathrm{GeV} . e$ and $\mu$ are produced through the leptonic $\tau$ decays, although a $\mu$ pair can appear directly through $h_{i} / P_{i}$ decay. With the chosen decay mode the $\tau$ multiplicity is considerable even though the $\tau$-identification efficiency is much lower $(\sim 50 \%)$ when compared to that of $e$ and $\mu(\gtrsim 95 \%)$. Occasionally highly collimated QCD jets can fake $\tau^{\text {had }}$ 's and, as a result, $\tau$ multiplicity exceeds the expected number of 4 . This faking, however, disappears with a higher $p_{\mathrm{T}}$ cut.

The $p_{\mathrm{T}}$ distributions of the leading and of the third leading lepton are shown in the bottom row of Fig. 1. It is evident that the leading lepton is energetic enough to trigger the event, should a single-lepton trigger be deployed. The rest of the leptons have sufficient $p_{\mathrm{T}}$ to be selected by a multilepton-based analysis, such as the ones developed by CMS [24] and ATLAS [25]. For instance, for the third leading $e, \mu$, and $\tau^{\text {had }}$, around $0.05,10$, and 43 events with $p_{\mathrm{T}}>10 \mathrm{GeV}$ are expected, respectively. Clearly, a multielectron signature is the least promising.

Such analyses, apart from the requirement of at least three or four leptons (including taus), require a high value of $E_{\mathrm{T}}^{\text {miss }}$ or of the scalar sum of reconstructed objects: leptons, jets and/or $E_{\mathrm{T}}^{\mathrm{miss}}$. For the chosen signal many 

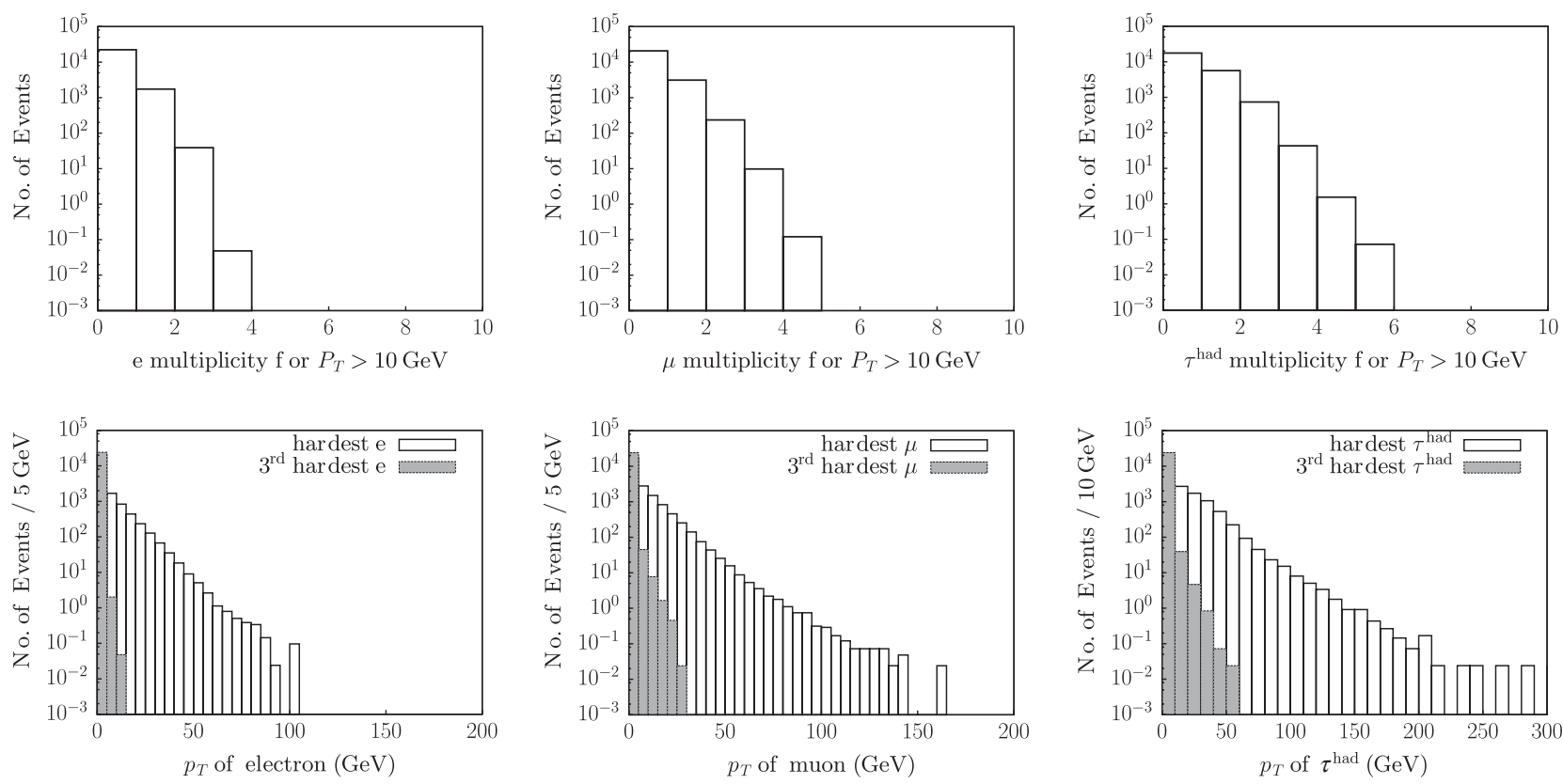

FIG. 1. Multiplicity (top row) for $e$ (left), $\mu$ (middle) and hadronically decaying $\tau$ (right) with $p_{\mathrm{T}}>10 \mathrm{GeV} . p_{\mathrm{T}}$ distributions (bottom row) for the leading (white) and the third leading (light grey) $e$ (left), $\mu$ (middle) and hadronically decaying $\tau$ (right). These plots correspond to $\sqrt{s}=8 \mathrm{TeV}$ with $\mathcal{L}=20 \mathrm{fb}^{-1}$.

neutrinos $(\gtrsim 6)$ appearing in the final state from $\tilde{\chi}_{4}^{0}$ and from $\tau$ decay give rise to moderately high missing transverse energy, $E_{\mathrm{T}}^{\text {miss }}$, as depicted in Fig. 2 (left). Besides $E_{\mathrm{T}}^{\text {miss }}$, the scalar sum of the $p_{\mathrm{T}}$ of all reconstructed leptons, $H_{\mathrm{T}}^{\ell}$, is also high in such events, as shown in Fig. 2 (right). Alternatively, the sum of $E_{\mathrm{T}}^{\text {miss }}$ and $H_{\mathrm{T}}^{\ell}$ can be deployed for further background rejection. These observables can provide additional handles when selecting events with many leptons. Also the invariant masses $m_{\ell^{+} \ell^{-}}$and $m_{2 \ell^{+}} 2 \ell^{-}$are useful for the purpose of signal distinction.

A word of caution is due here. In the discussion on multilepton analyses so far, prompt leptons are selected after imposing an upper limit on the transverse and the longitudinal impact parameters, in order to reject cosmicray muons and ensure good-quality track selection. Such a selection criterion should be relaxed or even reversed, if sensitivity to the $\mu \nu \mathrm{SSM}$ events is sought after. The reason stems from the long lifetime of the $\tilde{\chi}_{4}^{0}$ and hence the DV that its decay creates. This feature is quantified in the middle plot of Fig. 2, where the decay-length distribution is drawn. As expected from the proper decay length of $c \tau_{\tilde{\chi}_{4}^{0}} \approx 30 \mathrm{~cm}$, in a significant percentage of events, $\tilde{\chi}_{4}^{0}$ decays inside the tracker; e.g. $28 \%$ of events decay within $30 \mathrm{~cm}$ and $44 \%$ events within $1 \mathrm{~m}$. Therefore, the $\mu \nu \mathrm{SSM}$ signal events will be characterized by displaced $\tau$ leptons plus neutrinos. This distinctive signature opens up the possibility to exploit current or future variations of analyses carried out by ATLAS and CMS looking for a displaced muon and tracks [26] or searching for displaced dileptons [27] or muon jets [28] arising in Higgs decays to pairs of long-lived invisible particles.

The kinematics of the DVs and their products are demonstrated in Fig. 3. The $\tilde{\chi}_{4}^{0}$ boost, expressed by $\beta \gamma$, where $\beta$ is $\tilde{\chi}_{4}^{0}$ velocity over $c$ and $\gamma$ the Lorentz factor, versus the pseudorapidity $\eta$ is shown on the left. The shape reflects the fact that a single particle $\left(h_{4}\right)$ is produced at the hard
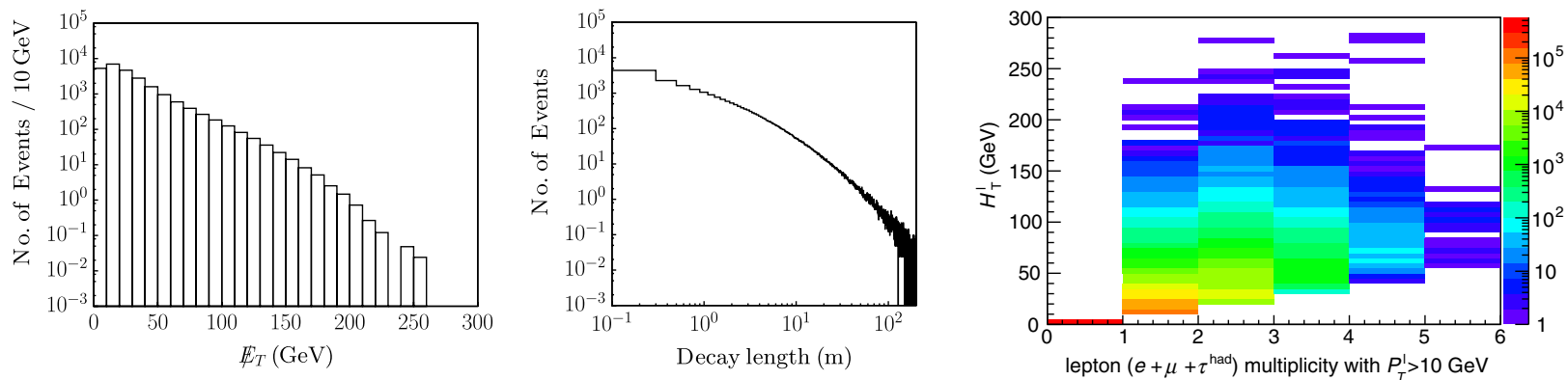

FIG. 2 (color online). $\quad \mathbb{E}_{T}$ distribution (left), $\tilde{\chi}_{4}^{0}$ decay-length distribution (middle) and $H_{\mathrm{T}}^{\ell}$ versus lepton multiplicity (right) for $\sqrt{s}=8 \mathrm{TeV}$ and $\mathcal{L}=20 \mathrm{fb}^{-1}$. 

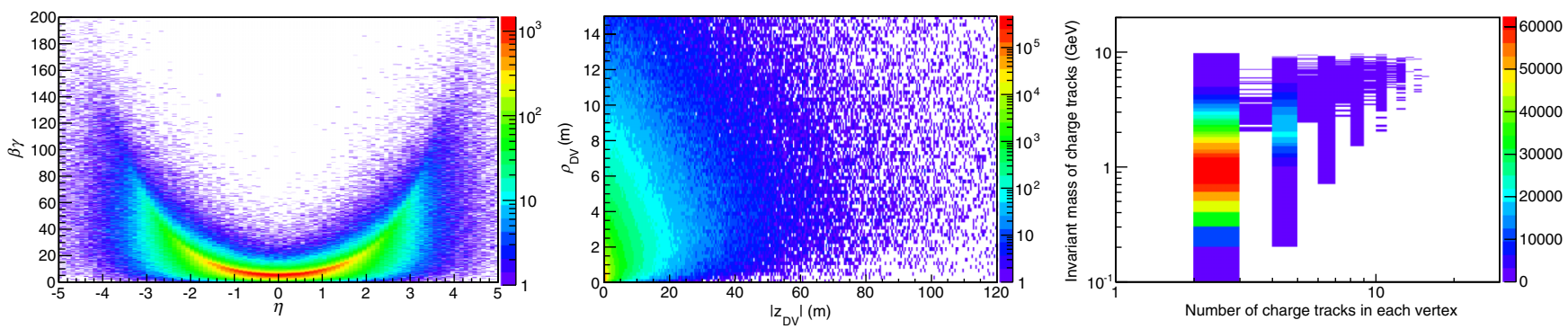

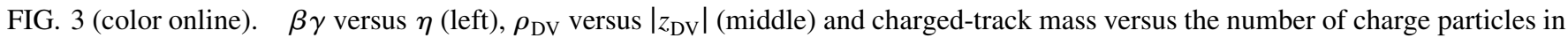
each vertex (right) for $\tilde{\chi}_{4}^{0}$ and for $\sqrt{s}=8 \mathrm{TeV}$ and $\mathcal{L}=20 \mathrm{fb}^{-1}$.

scattering of $p p$ collisions; hence, low momentum is expected in the central region. The average boost is comparable to the signal analyzed in an ATLAS search for a muon and tracks originating from DVs [26]. The boost affects the efficiency with which such a DV can be reconstructed, since high $\tilde{\chi}_{4}^{0}$ boost leads to collimated tracks that are difficult to differentiate from primary vertices.

In the middle plot in Fig. 3, the spacial distribution of a DV is displayed in cylindrical coordinates. A large fraction of DVs falls in the inner-tracker volume of a LHC experiment, i.e. $\rho_{\mathrm{DV}} \lesssim 1 \mathrm{~m}$ and $\left|z_{\mathrm{DV}}\right| \lesssim 2.5 \mathrm{~m}$; thus, DVs arising in the $\mu \nu \mathrm{SSM}$ should be detectable at the LHC, either with existing analyses [26-28] or via variations of those to search for displaced taus and $E_{\mathrm{T}}^{\text {miss }}$.

In the right panel of Fig. 3, we show the correlation between the number of charged tracks in each DV, $N_{\text {trk }}$, and their invariant mass $m_{\mathrm{DV}}$. A selection of high- $N_{\text {trk }}$ and high- $m_{\mathrm{DV}}$ has been demonstrated [26] to efficiently suppress background from long-lived SM particles ( $B$ mesons, kaons). The modulation observed in $N_{\text {trk }}$ is due to the oneprong or three-prong hadronic $\tau$ decays.

Summarizing, the $\mu \nu \mathrm{SSM}$ could be tested at the LHC through the production of a Higgs-like scalar with a mass about $125 \mathrm{GeV}$ which decays into a pair of light long-lived neutralinos. Such events could be probed by ATLAS and CMS with the currently available $8 \mathrm{TeV}$ data in two ways: either by looking for multilepton events produced in the SUSY cascade decay chain, when relaxing or even reversing the requirement for the leptons to come from the primary vertex, or by searching for tracks not pointing back to the primary vertex, originating from a secondary vertex. In either case, a moderately high missing transverse energy due to multiple neutrinos is expected. In principle, other Higgs boson decay chains or other processes might have been addressed to test the $\mu \nu \mathrm{SSM}$. However, we leave this necessary task for a future work [29].

P. G. thanks S. Biswas, K. Ghosh and C. B. Park for insightful discussions. The work of P.G. and C.M. was supported in part by the Spanish MINECO under Grants No. FPA2009-08958 and No. FPA2012-34694, and under the "Centro de Excelencia Severo Ochoa" Programme SEV-2012-0249, by the Comunidad de Madrid under Grant No. HEPHACOS S2009/ESP-1473, and by the European Union under the Marie Curie-ITN Program PITN-GA-2009-237920. The work of D. L. was supported by the Argentinian CONICET. The work of V.M. was supported by the Spanish MINECO under Grant No. FPA2009-13234-C04-01 and by the Spanish AECID under PCI Project No. A1/035250/11. The work of R. R. was supported by the Ramón y Cajal program of the Spanish MINECO and also thanks the support of the MINECO under Grant No. FPA2011-29678. The authors also acknowledge the support of the MINECO's Consolider-Ingenio 2010 Programme under Grant No. MultiDark CSD2009-00064.
[1] D. E. López-Fogliani and C. Muñoz, Phys. Rev. Lett. 97, 041801 (2006).

[2] N. Escudero, D. E. López-Fogliani, C. Muñoz, and R. Ruiz de Austri, J. High Energy Phys. 12 (2008) 099.

[3] S.P. Martin, arXiv:hep-ph/9709356.

[4] J. E. Kim and H. P. Nilles, Phys. Lett. 138B, 150 (1984).

[5] U. Ellwanger, C. Hugonie, and A. M. Teixeira, Phys. Rep. 496, 1 (2010).

[6] J. Fidalgo, D. E. López-Fogliani, C. Muñoz, and R. Ruiz de Austri, J. High Energy Phys. 08 (2009) 105.
[7] P. Ghosh and S. Roy, J. High Energy Phys. 04 (2009) 069.

[8] P. Ghosh, P. Dey, B. Mukhopadhyaya, and S. Roy, J. High Energy Phys. 05 (2010) 087.

[9] R. Barbier et al., Phys. Rep. 420, 1 (2005).

[10] H.-P. Nilles and N. Polonsky, Nucl. Phys. B484, 33 (1997).

[11] A. Bartl, M. Hirsch, A. Vicente, S. Liebler, and W. Porod, J. High Energy Phys. 05 (2009) 120.

[12] P. Bandyopadhyay, P. Ghosh, and S. Roy, Phys. Rev. D 84, 115022 (2011). 
[13] J. Fidalgo, D. E. López-Fogliani, C. Muñoz, and R. Ruiz de Austri, J. High Energy Phys. 10 (2011) 020.

[14] S. Liebler and W. Porod, Nucl. Phys. B855, 774 (2012).

[15] K.-Y. Choi, D. E. López-Fogliani, C. Muñoz, and R. R. Ruiz de Austri, J. Cosmol. Astropart. Phys. 03 (2010) 028; G. A. Gómez-Vargas, M. Fornasa, F. Zandanel, A. J. Cuesta, C. Muñoz, F. Prada, and G. Yepes, J. Cosmol. Astropart. Phys. 02 (2012) 001.

[16] D. J.H. Chung and A. J. Long, Phys. Rev. D 81, 123531 (2010).

[17] ATLAS Collaboration, Phys. Lett. B 716, 1 (2012).

[18] CMS Collaboration, Phys. Lett. B 716, 30 (2012).

[19] B. A. Dobrescu and K. T. Matchev, J. High Energy Phys. 09 (2000) 031; J. F. Gunion, D. Hooper, and B. McElrath, Phys. Rev. D 73, 015011 (2006).
[20] R. Dermisek and J. F. Gunion, Phys. Rev. Lett. 95, 041801 (2005).

[21] T. Sjostrand, S. Mrenna, and P.Z. Skands, J. High Energy Phys. 05 (2006) 026.

[22] LHC Higgs Cross Section Working Group, https:// twiki.cern.ch/twiki/bin/view/LHCPhysics/CrossSections.

[23] PGS4 web page, http://www.physics.ucdavis.edu/ conway/ research/software/pgs/pgs4-general.htm.

[24] CMS Collaboration, J. High Energy Phys. 06 (2012) 169.

[25] ATLAS Collaboration, J. High Energy Phys. 12 (2012) 124.

[26] ATLAS Collaboration, Phys. Lett. B 719, 280 (2013).

[27] CMS Collaboration, Report No. CMS-PAS-EXO-11-101, http://cdsweb.cern.ch/record/1456045.

[28] ATLAS Collaboration, Phys. Lett. B 721, 32 (2013).

[29] P. Ghosh et al. (unpublished). 